\title{
Stochastic Optimization of Power System Dynamics for Grid Resilience
}

\author{
Bryan Arguello \\ Sandia National Laboratories \\ barguel@sandia.gov
}

\author{
Nathan Stewart \\ Sandia National Laboratories \\ nastewa@sandia.gov
}

\author{
Matthew Hoffman \\ Sandia National Laboratories \\ mjhoffm@sandia.gov
}

\begin{abstract}
When faced with uncertainty regarding potential failure contingencies, prioritizing system resilience through optimal control of exciter reference voltage and mechanical torque can be arduous due to the scope of potential failure contingencies. Optimal control schemes can be generated through a two-stage stochastic optimization model by anticipating a set of contingencies with associated probabilities of occurrence, followed by the optimal recourse action once the contingency has been realized. The first stage, common across all contingency scenarios, co-optimally positions the grid for the set of possible contingencies. The second stage dynamically assesses the impact of each contingency and allows for emergency control response. By unifying the optimal control scheme prior and post the failure contingency, a singular policy can be constructed to maximize system resilience.
\end{abstract}

\section{Introduction}

In analyzing the behavior of the electric power grid after a major disruptive event, full dynamic models are critical. During such an event, real or reactive power, voltages, and other system metrics may go beyond limits enforced by steady state optimal power flow models (for general references in power system dynamics and stability, see [1,2]). Our work focuses on the problem of positioning the grid to be resilient to a set of possible outage scenarios through stochastic optimization over power system dynamics. In each scenario, we allow second stage recourse through generator control to improve grid stability after the outage.

The transient stability constrained optimal power flow (TSCOPF) problem, introduced in [3], optimizes power flow to minimize generation cost, while using power system dynamics to ensure stability under contingencies. Optimizing cost subject to stability constraints can leave the system close to constraint boundaries and vulnerable to additional perturbations; we instead incentivize safety margin, to keep the system farther from these boundaries. We are interested in stability under multiple possible contingencies, much like the multi-contingency transient stability constrained optimal power flow problem (MC-TSCOPF) introduced in [4]. Our research differs from MC-TSCOPF in that it 1) assumes the system is already at some (non-optimal) steady state prior to the contingency and explicitly addresses the dynamic control actions necessary to improve contingency readiness, and 2) additionally addresses post-contingency corrective control in the second stage. Our second-stage problem is similar to the transient stability emergency control (TSEC) problem (e.g., [5]) of ensuring grid stability after a contingency (e.g., by reducing generation and shedding load).

The three main techniques for solving TSCOPF and TSEC are direct approaches, single-machine equivalent (SIME) reduction, and computational intelligence. See [6] for a recent overview of these techniques. Most prevalent and flexible is the direct approach, which involves discretizing differential equations that express system dynamics. These discretized equations can be solved directly as part of the optimization (simultaneous discretization), in a simulation sub-problem (sequential discretization), or via a hybrid of the two. For expanded discussion of these methods and demonstration of the hybrid method, see [7]. While our problem formulation is agnostic of discretization approach, our results are obtained via simultaneous discretization. See [8] for enhanced discretization techniques.

Direct approaches are typically solved via an interior point method, and can benefit from reduction and parallelization techniques. For a reduced-space approach and parallelized reduced-space interior point method, see [9] and [10]. A GPU-based parallelization approach to solving TSCOPF is given in [11]. Metaheuristics have occasionally been used to solve these problems, include whale optimization [12], hybrid particle swarm, and artificial physics optimization[13].

Our optimization model prepares the grid for multiple contingencies before any one contingency 
occurs and optimizes stability after the contingency is realized. Our model does not directly consider economic generation cost in the objective function, as we focus on system resilience to major emergencies. Our formulation maximizes safety margins for voltage and frequency, to both ensure adequate power quality post-contingency and reduce the chance of a cascading failure. Unlike aforementioned TSCOPF research where power flow is optimized, we optimize directly over the exciter reference voltage and mechanical torque generator controls throughout the full time horizon. Furthermore, our research is the first to optimize grid dynamics in preparation for a multitude of possible contingencies, simultaneously with optimizing the post-contingency controls.

\section{Grid Resilience and Stability Model}

We use the fourth-order flux decay generator model and turbine with no reheating model from [1]. For realism, loads can also be modeled dynamically [14]; the importance of dynamic load modeling compared to static load modeling is investigated in [15]. Our power system model uses the exponential recovery dynamic load model [16]. Our model is a nonlinear two-stage stochastic differential algebraic equation (DAE) formulation. The differential equations of the generators and loads can be discretized through any available discretization scheme including finite differences and collocation. We use a set of contingencies such as line faults and generator failures that occur at the same time $t_{f}$ to form our scenarios. The first stage varies generator controls (exciter reference voltages and mechanical torque) before $t_{f}$ so that the grid is best prepared for any scenario to occur. Meanwhile, the second stage varies generator controls after $t_{f}$ in each scenario to maximize grid stability. The generator controls (indirectly) adjust generator real and reactive power output.

In the following sections, we detail our formulation by giving nomenclature, DAE equations, a description of discretization, stability objectives, and finally our two-stage stochastic programming model.

\subsection{Sets}

\begin{tabular}{lll} 
Set & Index Symbol & Description \\
\hline $\mathcal{B}$ & $\mathrm{b}$ & Buses \\
$\mathcal{G}$ & $\mathrm{g}$ & Generators \\
$\mathcal{L}$ & 1 & Loads \\
$\Psi$ & $\psi$ & Scenarios
\end{tabular}

\subsection{Indexed Sets}

\begin{tabular}{lll} 
Set & Index Symbol & Description \\
\hline $\mathcal{G}_{b}$ & $\mathrm{~b}$ & Generators at bus b \\
$\mathcal{L}_{b}$ & $\mathrm{~b}$ & Loads at bus b
\end{tabular}

\subsection{DAE Variables}

\begin{tabular}{lll} 
Variable & Index & Description \\
\hline$\delta$ & $\mathrm{g}$ & Rotor angle \\
$\omega$ & $\mathrm{g}$ & Generator frequency \\
$E_{q}^{\prime}$ & $\mathrm{g}$ & q-axis transient voltage \\
$E_{f d}$ & $\mathrm{~g}$ & Field voltage \\
$I_{q}$ & $\mathrm{~g}$ & q-axis current \\
$I_{d}$ & $\mathrm{~g}$ & d-axis current \\
$T_{M}$ & $\mathrm{~g}$ & Shaft mechanical torque \\
$V$ & $\mathrm{~b}$ & Voltage \\
$\theta$ & $\mathrm{b}$ & Phase angle \\
$P_{L}$ & 1 & Active load power draw \\
$Q_{L}$ & 1 & Reactive load power draw \\
$x_{p}$ & 1 & Load active power state variable \\
$x_{q}$ & 1 & Load reactive power state variable
\end{tabular}

\subsection{Control Variables}

\begin{tabular}{lll} 
Variable & Index & Description \\
\hline$V_{\text {ref }}$ & $\mathrm{g}$ & Exciter reference voltage \\
$P_{\text {ref }}$ & $\mathrm{g}$ & Generator mechanical torque power
\end{tabular}

\subsection{Parameters}

\begin{tabular}{lll} 
Parameter & Index & Description \\
\hline $\mathcal{T}$ & & Time horizon \\
$\omega_{s}$ & & Rated synchronous speed \\
$M$ & $\mathrm{~g}$ & Shaft inertial constant \\
$D$ & $\mathrm{~g}$ & Damping coefficient \\
$K_{A}$ & $\mathrm{~g}$ & Exciter amplifier gain \\
$T_{A}$ & $\mathrm{~g}$ & Exciter amplifier time constant \\
$R_{s}$ & $\mathrm{~g}$ & Scaled resistance after dq \\
& & transformation \\
$X_{q}$ & $\mathrm{~g}$ & q-axis synchronous reactance \\
$X_{d}$ & $\mathrm{~g}$ & d-axis synchronous reactance \\
$X_{d}^{\prime}$ & $\mathrm{g}$ & d-axis transient reactance \\
$T_{d o}^{\prime}$ & $\mathrm{g}$ & Transient time constant \\
$T_{c h}$ & $\mathrm{~g}$ & Mechanical torque damping const. \\
$b_{g}$ & $\mathrm{~g}$ & Bus connected to generator $\mathrm{g}$ \\
$P o_{L}$ & 1 & Initial active power \\
$Q o_{L}$ & 1 & Initial reactive power \\
$T p_{L}$ & 1 & Active power time constant \\
$T q_{L}$ & 1 & Reactive power time constant \\
$\alpha_{s}$ & 1 & First active power exponent
\end{tabular}




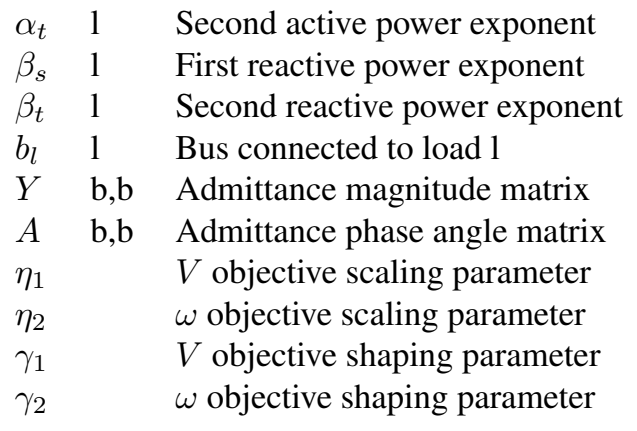

\subsection{DAE Equations}

Generator model:

$$
\begin{aligned}
& \frac{d \delta_{g}}{d t}=\omega_{g}-\omega_{s} \\
& \frac{d \omega_{g}}{d t}=\frac{T_{M_{g}}}{M_{g}}-E_{q_{g}}^{\prime} \frac{I_{q_{g}}}{M_{g}}-I_{d_{g}} I_{q_{g}} \frac{X_{q_{g}}-X_{d_{g}}^{\prime}}{M_{g}} \\
& \quad-D_{g} \frac{\omega_{g}-\omega_{s}}{M_{g}} \\
& \frac{d E_{q_{g}}^{\prime}}{d t}=-\frac{E_{q_{g}}}{T_{d_{g}}^{\prime}}-X_{d_{g}}-I_{d_{g}} \frac{X_{d_{g}}^{\prime}}{T_{d o_{g}}^{\prime}}+\frac{E_{f d_{g}}}{T_{d o_{g}}^{\prime}} \\
& \frac{d E_{f d_{g}}}{d t}=-\frac{E_{f d_{g}}}{T_{A_{g}}}+\left(V_{\text {refg }}-V_{b_{g}}\right) \frac{K_{A_{g}}}{T_{A_{g}}}
\end{aligned}
$$

$\forall g \in \mathcal{G}$

Governor model:

$\frac{d T_{M_{g}}}{d t}=\frac{P_{r e f_{g}}-T_{M_{g}}}{T_{c h_{g}}}$

$\forall g \in \mathcal{G}$

Stator equations:

$V_{b_{g}} \sin \left(\delta_{g}-\theta_{b_{g}}\right)+R_{s_{g}} I_{d_{g}}-X_{q_{g}} I_{q_{g}}=0$

$E_{q_{g}}^{\prime}-V_{b_{g}} \cos \left(\delta_{g}-\theta_{b_{g}}\right)-R_{s_{g}} I_{q_{g}}-X_{q_{g}}^{\prime} I_{d_{g}}=0$

$\forall g \in \mathcal{G}$
Exponential recovery load model:

$$
\begin{aligned}
& \frac{d x_{p_{l}}}{d t}=\frac{x_{p_{l}}}{T p_{L_{l}}}+P o_{L_{l}} V_{b_{l}}^{\alpha_{s_{l}}}-P o_{L_{l}} V_{b_{l}}^{\alpha_{t_{l}}} \\
& \frac{d x_{q_{l}}}{d t}=\frac{x_{q_{l}}}{T q_{L_{l}}}+Q o_{L_{l}} V_{b_{l}}^{\beta_{s_{l}}}-Q o_{L_{l}} V_{b_{l}}^{\beta_{t_{l}}} \\
& P_{L_{l}}=\frac{x_{p_{l}}}{T p_{L_{l}}}+P o_{L_{l}} V_{b_{l}}^{\alpha_{t_{l}}} \\
& Q_{L_{l}}=\frac{x_{q_{l}}}{T q_{L_{l}}}+Q o_{L_{l}} V_{b_{l}}^{\beta_{t_{l}}} \\
& \forall l \in \mathcal{L}
\end{aligned}
$$

Balance equations:

$$
\begin{array}{r}
-\sum_{i \in \mathcal{B}}\left(V_{i} V_{b} Y_{i, b} \cos \left(\theta_{i}-\theta_{b}-A_{i, b}\right)\right) \\
-\sum_{l \in \mathcal{L}_{b}}\left(P_{L_{l}}\right)+\sum_{g \in \mathcal{G}_{b}}\left(I_{d_{g}} V_{b} \sin \left(\delta_{g}-\theta_{b}\right)\right. \\
\left.\quad+I_{q_{g}} V_{b} \cos \left(\delta_{g}-\theta_{b}\right)\right)=0 \\
-\sum_{i \in \mathcal{B}}\left(V_{i} V_{b} Y_{i, k} \sin \left(\theta_{i}-\theta_{b}-A_{i, b}\right)\right) \\
-\sum_{l \in \mathcal{L}_{b}}\left(Q_{L_{l}}\right)+\sum_{g \in \mathcal{G}_{b}}\left(I_{d_{g}} V_{g} \cos \left(\delta_{g}-\theta_{b}\right)\right. \\
\left.+I_{q_{g}} V_{b} \sin \left(\delta_{g}-\theta_{b}\right)\right)=0
\end{array}
$$

$\forall b \in \mathcal{B}$

In addition we add two inequality constraints that limit the rate of change of $V_{\text {ref }}$ and $P_{\text {ref }}$. We do this to prevent solutions with highly oscillatory control choices, as this would not be replicable in reality.

$$
\begin{aligned}
& \left|\frac{d V_{r e f_{g}}}{d t}\right| \leq k \\
& \left|\frac{d P_{r e f_{g}}}{d t}\right| \leq k
\end{aligned}
$$

$\forall g \in \mathcal{G}$

Note that $k$ could be set differently for $V_{\text {ref }}$ and $P_{\text {ref }}$ or even uniquely for each generator. In practice, this value would be tied to the generator ramp rate of the system. A rather aggressive value of $k=3$ is used for this proof of concept. 


\subsection{Discretization}

To approximate the differential equations with algebraic equations, we first partition the time horizon $[0, T]$ through a finite set of points $\mathcal{P}=$ $\left\{0, t_{2}, \ldots, t_{f}, \ldots, t_{n-1}, T\right\}$ that includes the failure time. Common choices of $\mathcal{P}$ include uniform spacing and a scheme which adds more points around time periods where total variation is expected to be high such as a neighborhood after $t_{f}$. Next, $\mathcal{P}$ is used to discretize differential equations through a discretization scheme such as finite difference or collocation. As an example, the forward finite difference scheme approximates every derivative $\frac{d x}{d t}$ through the forward finite difference

$$
\frac{d x}{d t} \approx \frac{x_{t_{k}}-x_{t_{k-1}}}{t_{k}-t_{k-1}}
$$

After every derivative in the DAE is approximated with a finite difference, the DAE is approximated through a system of algebraic equations. Henceforth, we refer to a discretized approximation of DAE (1) - (15) as (1)' (15)'.

Note that numerical circumstances around dynamic simulations are different than those around solving our DAE model. With dynamic simulation, numerical inaccuracies are propogated forward in time as the simulation progresses. DAE models are used to obtain a full system solution over the full time horizon simultaneously.

\subsection{Stability Metrics}

Upon discretization, discrete values of bus voltage and angular velocity are available to form stability metrics for various types of grid stability.

$$
M_{v}\left(t_{1}, t_{2}\right)=\sum_{t \in\left\{\tau \in \mathcal{P} \mid t_{1} \leq \tau<t_{2}\right\}} \sum_{b \in \mathcal{B}}\left(\frac{1-V_{b, t}}{\eta_{1}}\right)^{\gamma_{1}}
$$

measures how far buses deviate from a nominal voltage of 1 p.u. between $t_{1}$ and $t_{2}$. Similarly,

$$
M_{\omega}\left(t_{1}, t_{2}\right)=\sum_{t \in\left\{\tau \in \mathcal{P} \mid t_{1} \leq \tau<t_{2}\right\}} \sum_{g \in \mathcal{G}}\left(\frac{\omega_{g, t}-\omega_{s}}{\omega_{s} \cdot \eta_{2}}\right)^{\gamma_{2}}
$$

measures how far frequency deviates from nominal frequency between $t_{1}$ and $t_{2}$. The parameters $\eta$ and $\gamma$ can be used to scale and shape the stability metrics.
Penalizing these deviation metrics both incentivizes improved power quality, and increases margin against over/under voltage and frequency limits to reduce the likelihood of protective tripping if any follow-on contingencies occur. Prioritizing voltage and frequency deviation should maximize grid stability in the short term given a failure contingency. While these are sufficient for proof of concept, additional stability metrics such as transient stability can be included.

We later add these stability metrics together to form our model's objective function.

\subsection{Stochastic Model}

From the discretized dynamic model of the power grid, we construct a two stage stochastic program with recourse. This mathematical program takes the form

$$
\begin{aligned}
& \min _{x, y_{\psi}} c(x)+E\left[d\left(y_{\psi}\right)\right] \\
& \text { s.t. } \\
& \qquad f(x) \leq b \\
& \quad g_{\psi}\left(y_{\psi}\right) \leq f_{\psi} \\
& \qquad h(x)+k\left(y_{\psi}\right) \leq g_{\psi}
\end{aligned}
$$$$
\forall \psi \in \Psi
$$

$\forall \psi \in \Psi$

This allows only choices of $x$ that leave constraints (23) feasible. Within these constraints, $x$ is chosen, and $y_{\psi}$ is chosen for every scenario $\psi$, so that the first stage objective $c(x)$ and expected value of second stage objectives $d\left(y_{\psi}\right)$ are optimal. See [17] for further details on stochastic programming.

Unlike most stochastic programming models in the literature, our model's first stage and second stage are separated by time. Our first stage decisions are made for $t \in\left[0, t_{f}\right)$ and prepares the grid to be in a resilient state before any contingency occurs. Meanwhile our second stage decisions are made for $t \in\left[t_{f}, T\right]$ and stabilizes the grid after any contingency is realized.

The first stage of our stochastic model optimizes control variables $V_{\text {ref }}$ and $P_{\text {ref }}$ over $\left[0, t_{f}\right)$ to prepare for any of the scenarios in $\Psi$. Therefore our first stage constraints - effectively (21) - are equations (1)' - (15)' discretized only for $t \in\left\{\tau \in \mathcal{P} \mid \tau<t_{f}\right\}$.

Our model's second stage optimizes control variables $V_{\text {ref }}$ and $P_{\text {ref }}$ to maximize stability in each scenario. For every $\psi \in \Psi$, we add equations (1)' (15)' discretized only for $t \in\left\{\tau \in \mathcal{P} \mid \tau \geq t_{f}\right\}$ as our second stage constraints (22).

To ensure continuity of the dynamic model between $\left[0, t_{f}\right)$ and $\left[t_{f}, T\right]$, we add the single discretized constraint for (1)' - (5)', (8)' - (9)' pertinent to $t_{f}$ and the time period before it. Note that these constraints 
form (23) above; they contain second stage decision variables for $t_{f}$ and first stage decision variables for the time before it.

Finally, using our stability metrics $M_{v}$ and $M_{\omega}$, our objective (19) is

$$
\begin{aligned}
& \min _{V_{r e f}, P_{r e f}} M_{v}\left(0, t_{f}\right)+M_{\omega}\left(0, t_{f}\right)+ \\
& \sum_{\psi \in \Psi} p_{\psi}\left(M_{v}\left(t_{f}, T\right)+M_{\omega}\left(t_{f}, t\right)\right)
\end{aligned}
$$

Note that the first two terms use $V$ and $\omega$ variables from the first stage equations while the final terms use $V$ and $\omega$ variables from the various second stage scenario equations.

In effect, our model optimizes over a singular set of controls with the anticipation of various potential failure contingency scenarios - each with its own probability occurring. The model then optimizes controls over every scenario to maximize stability in the event that scenario occurs.

Note that in general, the first stage could actually precede the second stage by minutes or more. In that case, it may be prudent to incorporate a cost incentive in the first stage to prevent economically unrealistic results. For this proof of concept, however, we are focused on the stability problem, and present the stages as temporally contiguous and omit a cost penalty for simplicity.

We consider simple load, line, and generator trips as our contingencies. For ease of exposition, we define the post-failure partition $\mathcal{P}_{f}=\left\{t \in \mathcal{P} \mid t \geq t_{f}\right\}$.

To model a load trip we deactivate the differential and algebraic load constraints (8)' - (11)' and fix $P_{L}$, $Q_{L}, x p_{L}, x q_{L}$ to 0 for $t \in \mathcal{P}_{f}$.

A line trip eliminates the connection between two buses $b_{1}$ and $b_{2}$. This is done by setting $Y_{b_{1}, b_{2}}$ and $Y_{b_{2}, b_{1}}$ to zero and then recalculating the diagonal entries $Y_{b_{1}, b_{1}}$ and $Y_{b_{2}, b_{2}}$ for $t \in \mathcal{P}_{f}$. Note that in our implementation, $Y$ includes time as an additional index to allow line trips.

Finally, for generator trips, constraints (6)' - (7)' are deactivated and variables $I_{q}, I_{d}$ are fixed to zero for $t \in$ $\mathcal{P}_{f}$. This prevents generator current from entering the power network.

When performing single-scenario experiments with these failure contingencies, we are assuming that the failure happens deterministically, and that we optimize the stability given this future failure. If we perform a multi-scenario experiment, however, we need to introduce uncertainty in the likelihood of each scenario occurring. We define $p_{\psi}$ as the probability that contingency scenario $\psi$ occurs.

\section{Experimentation and Results}

We demonstrate our model through experiments on the WSCC 9-bus power system. Our experiments optimize grid resilience and stability for different failure contingencies.

\subsection{Solution Methodology}

Our model is developed in the mathematical programming language Pyomo [18]. Pyomo's sublanguage Pyomo.DAE facilitates expression of differential equations and has automatic discretization capability with various optional discretization schemes. The experiments described in this section use uniform spacing and collocation techniques implemented in Pyomo.DAE.

Pyomo transforms our DAE model into a nonlinear program and can interface with most nonlinear solvers. In our experiments, we used both IPOPT [19] and Knitro [20].

\subsection{No-Failure Deterministic Case}

Let us first consider a single scenario with no failures. Solving our model with shape parameters $\eta=1$ and $\gamma=2$, a time horizon $T=[0,3]$, and 40 discretized points, we get the following results. Figures $1,2,3$, and 4 depict $V, \omega, V_{\text {ref }}$, and $P_{\text {ref }}$ respectively over time.

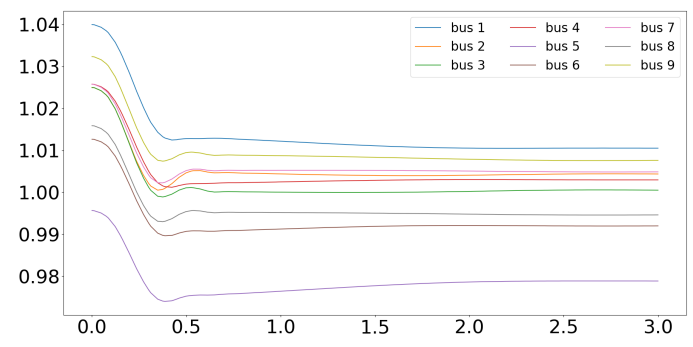

Figure 1. $V$ (p.u) vs. $t$ with no failure

Note that although the system is initially in a steady state, it quickly moves away from that steady state (via generator controls) towards a state with more safety margin. We can see that even without failures, the system can benefit from controls - here, primarily to improve voltage quality.

Of note, the frequency separation depicted in Figure 2 is both very small and brief, and not of stability concern (it is causing the generator rotor angles to converge, not diverge). 


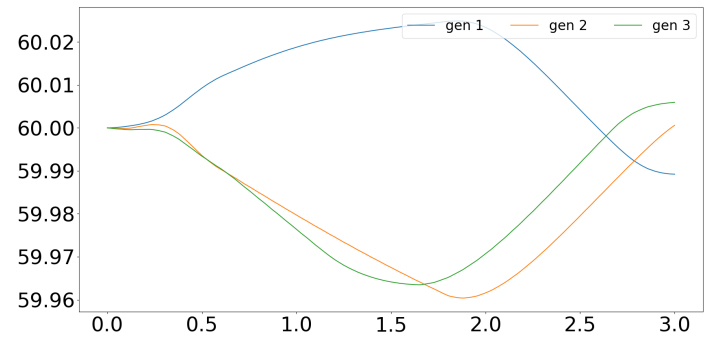

Figure 2. $\omega$ (Hz.) vs. $t$ with no failure

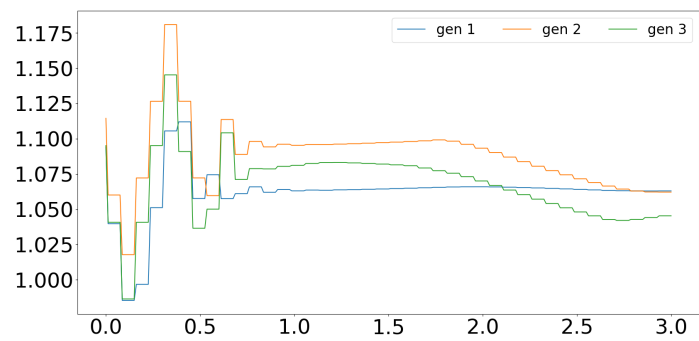

Figure 3. $V_{r e f}$ vs. $t$ control trajectory with no failure

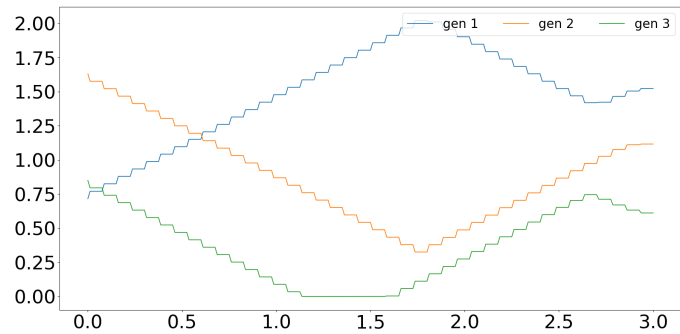

Figure 4. $P_{r e f}$ vs. $t$ control trajectory with no failure

\subsection{Deterministic Failure Cases}

At $t_{f}=1.5$, we induce a line trip between buses 5 and 7 , a generator trip at bus 1 , and a load trip at bus 5 . Our model yields the following voltage responses when dealing with each scenario individually. As the first stage is effectively allowed to anticipate each upcoming failure, these results show the (unrealistic) best possible preventive-corrective control actions for each scenario.

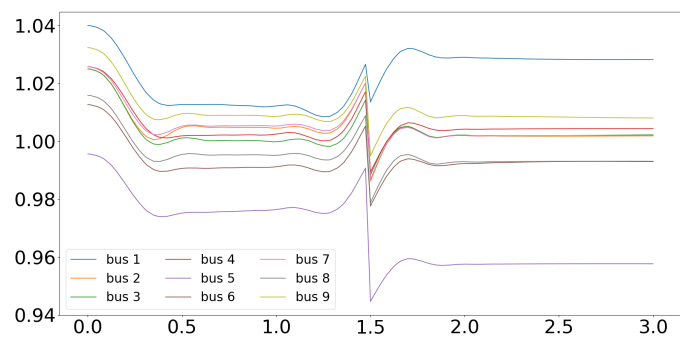

Figure 5. $\quad V$ vs. $t$ with line 5-7 trip at $t_{f}=1.5$

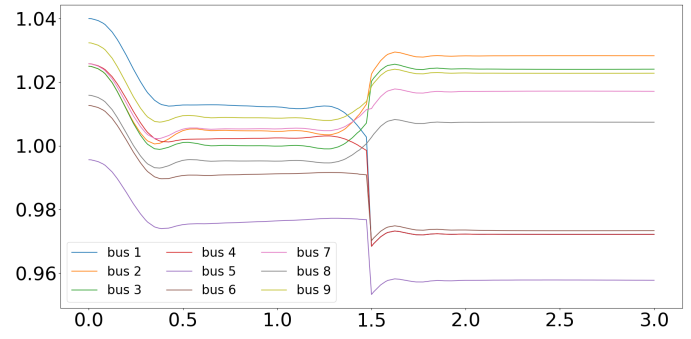

Figure 6. $V$ vs. $t$ with generator trip at Bus 1

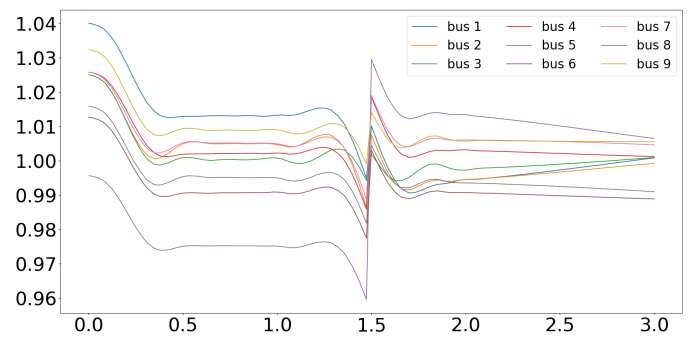

Figure 7. $V$ vs. $t$ with load trip at Bus $\mathbf{5}$

For this small system, even single-component contingencies are a major perturbation to the system. Nonetheless, coupling preventive control (before $t_{f}$ ) and corrective control (after $t_{f}$ ) allows the system to stabilize the system fairly quickly. Naturally, behavior may take significantly longer to stabilize using more realistic ramp rates and bounds on control variables.

In the following subsection we show the results when the model can not anticipate which failure will occur.

\subsection{Multi-Scenario Stochastic Case}

Here we optimize the first stage over several scenarios, with scenario-specific dynamics and recourse control in the second stage. In this example there are 4 scenarios of equal probability: the three failure events in Section 3.3 plus a no-failure scenario. This multi-scenario experiment yields the following first-stage results. Note that all first-stage dynamics are due to control actions (to improve upon the non-optimal initial condition and prepare for contingencies).

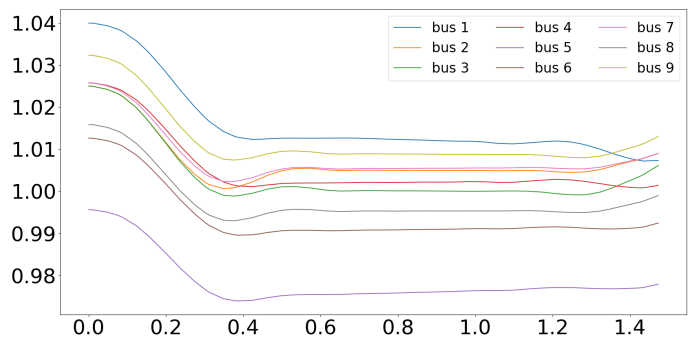

Figure 8. First stage $V$ vs. $t$ 


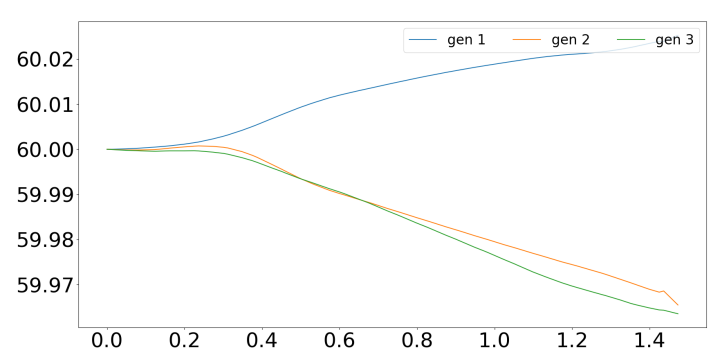

Figure 9. First stage $\omega$ vs. $t$

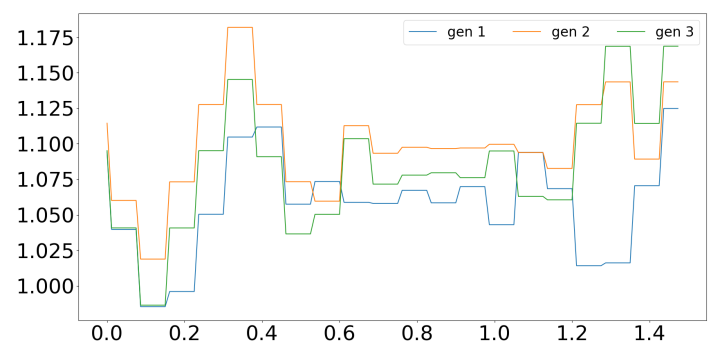

Figure 10. First stage $V_{r e f}$ vs. $t$ control

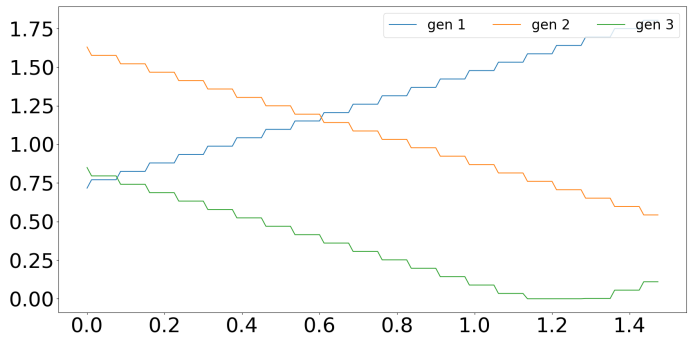

Figure 11. First stage $P_{r e f}$ vs. $t$ control

While the frequency separation in Figure 9 would not be sustainable over longer time windows, in this case it optimally pre-positions the grid for the possible set of upcoming failures (and although not shown here, is corrected quickly after each failure).

Figures 12-15 show the voltage trajectory for each scenario across the entire time horizon. Note that the pre-contingency behavior is the same as Figure 8 across all scenarios.

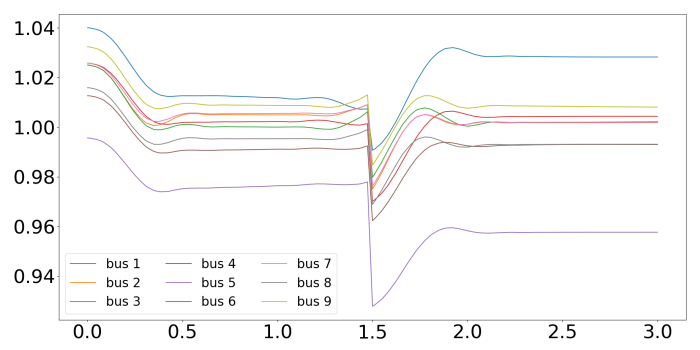

Figure 12. $V$ vs. $t$ for line 5-7 trip

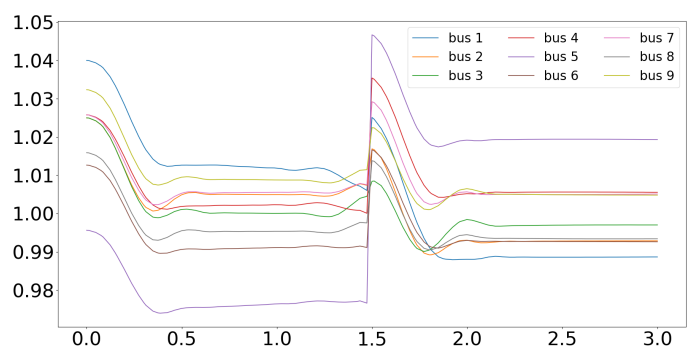

Figure 13. $\quad V$ vs. $t$ for load trip at Bus 5

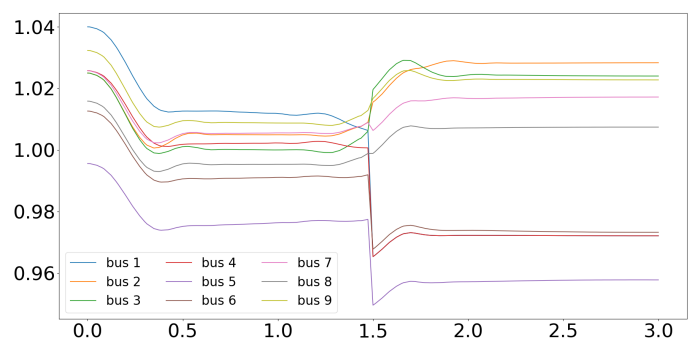

Figure 14. $V$ vs. $t$ for generator trip at Bus 1

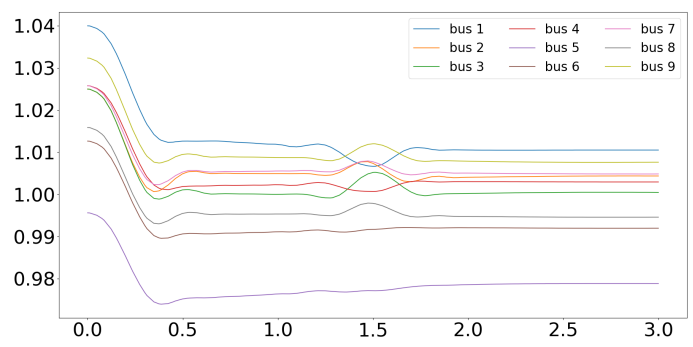

Figure 15. $\quad V$ vs. $t$ with no failures

As these figures show, the optimization is able to keep voltage within desired values (except for a brief excursion at bus 5 in Figure 12). Frequency was also kept within $0.2 \%$ of nominal in all cases.

Figure 14 is particularly interesting; while the voltages diverge, they are kept centered within reasonable bounds around 1.0 p.u. despite the sudden loss of $24 \%$ of total generation. This is significant; although not shown here, without control the system experiences major voltage drop across the entire system. Coupling first and second-stage controls is particularly effective, yielding a $65 \%$ reduction in objective value compared to pre-positioning alone, or $61 \%$ reduction compared to recourse control alone.

While the results shown here are optimistic due to relaxed rate-of-change and bound constraints, they provide proof of concept that the combined pre-positioning and emergency control formulation can provide significant benefit during emergencies. 
Furthermore, they demonstrate that it is conceivable to pre-position against multiple serious contingencies whose systemic effects are considerably different. However, such pre-positioning is likely to be far from optimal in nominal operating conditions (economic penalties may mitigate this somewhat, albeit at some cost to contingency readiness). In Figure 16 we see that significant control actions are required for the no-failure case to "stand down" from contingency readiness.

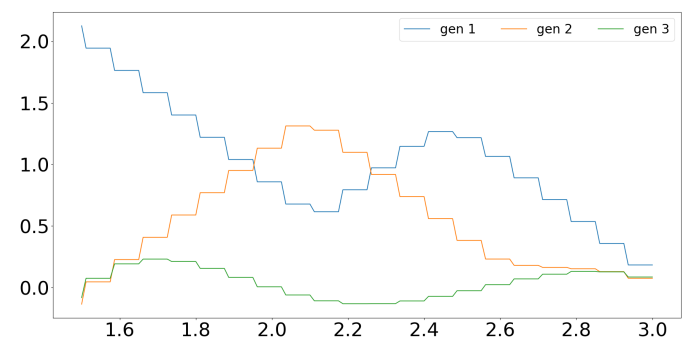

Figure 16. $P_{r e f}$ vs. $t>t_{f}$ with no failures

\section{Conclusion}

We have demonstrated the ability to solve our two-stage stochastic optimization model with dynamic constraints that represent power system dynamics. This class of problem is important to power system resilience, as 1) both preparation (first stage) and emergency control (second stage) are needed to ensure system operability under severe emergencies, 2) coupling these decisions together in the same framework should allow better solutions across a wider set of contingencies, and 3) system dynamics are critical to assessing grid stability in such emergencies. Additionally, motivated by our intended use on severe emergency cases, we demonstrate the use of power stability and quality objectives, as opposed to economic objectives typical in the literature. This framework allows preparation against an uncertain space of contingencies scenarios, as well as immediate recourse control once the uncertainty is realized. This provides the greatest margin for safety against substantial voltage and frequency deviations.

\subsection{Future Research}

Our current work demonstrates proof of concept on the WSCC 9-bus system. Future research will look at scalability to larger systems and scenario sets, and effectiveness against multi-component failure scenarios. Load shed will be added as a second-stage decision variable to improve feasibility in such cases. Control of inverter-based resources may be of significant benefit as well. Higher temporal fidelity and/or more sophisticated temporal discretization may be needed to accurately capture severe post-contingency behavior.

In cases where any excursions outside bounds are unacceptable, hard constraints can be set. For example, to ensure transient stabiilty, rotor angle should be no more than $100^{\circ}$ from system center of inertia. Likewise, voltage and frequency bound constraints would ensure that the solutions do not exceed bounds that would cause protective tripping in the real system. Additional objective penalties and/or constraints could be considered (e.g., voltage stability, small signal stability, and transmission line power limits) to provide more assurance of system stability in severe contingencies.

\section{Acknowledgments}

We extend our warm appreciation to Bethany Nicholson for her assistance with Pyomo modeling and discretization questions.

Sandia National Laboratories is a multimission laboratory managed and operated by National Technology \& Engineering Solutions of Sandia, LLC, a wholly owned subsidiary of Honeywell International Inc., for the U.S. Department of Energy's National Nuclear Security Administration under contract DE-NA0003525. SAND2020-13081 C.

This paper describes objective technical results and analysis. Any subjective views or opinions that might be expressed in the paper do not necessarily represent the views of the U.S. Department of Energy or the United States Government.

\section{References}

[1] P. W. Sauer, M. A. Pai, and J. H. Chow, Power system dynamics and stability: with synchrophasor measurement and power system toolbox. John Wiley \& Sons, 2017.

[2] P. Kundur, N. J. Balu, and M. G. Lauby, Power system stability and control, vol. 7. McGraw-hill New York, 1994.

[3] D. Gan, R. J. Thomas, and R. D. Zimmerman, "Stability-constrained optimal power flow," IEEE Transactions on Power Systems, vol. 15, no. 2, pp. 535-540, 2000.

[4] Y. Yuan, J. Kubokawa, and H. Sasaki, "A solution of optimal power flow with multicontingency transient stability constraints," IEEE Transactions on Power Systems, vol. 18, no. 3, pp. 1094-1102, 2003.

[5] Z. Li, G. Yao, G. Geng, and Q. Jiang, "An efficient optimal control method for open-loop transient stability emergency control," IEEE Transactions on Power Systems, vol. 32, no. 4, pp. 2704-2713, 2016.

[6] S. Abhyankar, G. Geng, M. Anitescu, X. Wang, and V. Dinavahi, "Solution techniques for transient stability-constrained optimal power flow-part i," IET 
Generation, Transmission \& Distribution, vol. 11, no. 12, pp. 3177-3185, 2017.

[7] G. Geng, V. Ajjarapu, and Q. Jiang, "A hybrid dynamic optimization approach for stability constrained optimal power flow," IEEE Transactions on Power Systems, vol. 29, no. 5, pp. 2138-2149, 2014.

[8] Q. Jiang and Z. Huang, "An enhanced numerical discretization method for transient stability constrained optimal power flow," IEEE Transactions on Power Systems, vol. 25, no. 4, pp. 1790-1797, 2010.

[9] Q. Jiang and G. Geng, "A reduced-space interior point method for transient stability constrained optimal power flow," IEEE Transactions on Power Systems, vol. 25, no. 3, pp. 1232-1240, 2010.

[10] G. Geng and Q. Jiang, "A two-level parallel decomposition approach for transient stability constrained optimal power flow," IEEE Transactions on Power Systems, vol. 27, no. 4, pp. 2063-2073, 2012.

[11] G. Geng, Q. Jiang, and Y. Sun, "Parallel transient stability-constrained optimal power flow using gpu as coprocessor," IEEE Transactions on Smart Grid, vol. 8, no. 3, pp. 1436-1445, 2016.

[12] D. Prasad, A. Mukherjee, G. Shankar, and V. Mukherjee, "Application of chaotic whale optimisation algorithm for transient stability constrained optimal power flow," IET Science, Measurement \& Technology, vol. 11, no. 8, pp. 1002-1013, 2017.

[13] K. Teeparthi and D. V. Kumar, "Dynamic power system security analysis using a hybrid pso-apo algorithm," Engineering, Technology \& Applied Science Research, vol. 7, no. 6, pp. 2124-2131, 2017.

[14] D. Karlsson and D. J. Hill, "Modelling and identification of nonlinear dynamic loads in power systems," IEEE Transactions on Power Systems, vol. 9, no. 1, pp. 157-166, 1994.

[15] R. Zhang, Y. Xu, W. Zhang, Z. Y. Dong, and Y. Zheng, "Impact of dynamic models on transient stability-constrained optimal power flow," in 2016 IEEE PES Asia-Pacific Power and Energy Engineering Conference (APPEEC), pp. 18-23, IEEE, 2016.

[16] D. J. Hill, "Nonlinear dynamic load models with recovery for voltage stability studies," IEEE transactions on power systems, vol. 8, no. 1, pp. 166-176, 1993.

[17] J. R. Birge and F. Louveaux, Introduction to stochastic programming. Springer Science \& Business Media, 2011.

[18] "Pyomo documentation 5.7," $2017 . \quad$ Available at https://pyomo.readthedocs.io/en/ stable/.

[19] "Ipopt documentation." Available at https:// coin-or.github.io/Ipopt/.

[20] "Knitro - artelys." Available at https://www.artelys.com/solvers/knitro/.

[21] C. Ledesma, Calle and Arredondo, "Multi-contingency tscopf based on full-system simulation," IET Generation, Transmission \& Distribution, vol. 11, no. 1, pp. 64-72, 2017.

[22] H. Nguyen-Duc, L. Tran-Hoai, and D. V. Ngoc, "A novel approach to solve transient stability constrained optimal power flow problems," Turkish Journal of Electrical Engineering \& Computer Sciences, vol. 25, no. 6, pp. 4696-4705, 2017.
[23] H. Nguyen-Duc, L. Tran-Hoai, and H. Cao-Duc, "An approach to solve transient stability-constrained optimal power flow problem using support vector machines," Electric Power Components and Systems, vol. 45, no. 6, pp. 624-632, 2017.

[24] Y. Yang, W. Liu, J. Deng, H. Liu, H. Wei, and T. Wang, "A parallel approach for multi-contingency transient stability constrained optimal power flow," in 2017 IEEE Power \& Energy Society General Meeting, pp. 1-5, IEEE, 2017.

[25] Z. Li, G. Geng, and Q. Jiang, “Transient stability emergency control using asynchronous parallel mixed-integer pattern search," IEEE Transactions on Smart Grid, vol. 9, no. 4, pp. 2976-2985, 2016.

[26] M. Paramasivam, A. Salloum, V. Ajjarapu, V. Vittal, N. B. Bhatt, and S. Liu, "Dynamic optimization based reactive power planning to mitigate slow voltage recovery and short term voltage instability," IEEE Transactions on Power Systems, vol. 28, no. 4, pp. 3865-3873, 2013.

[27] G. C. Zweigle and V. Venkatasubramanian, "Wide-area optimal control of electric power systems with application to transient stability for higher order contingencies," IEEE Transactions on Power Systems, vol. 28, no. 3, pp. 2313-2320, 2013.

[28] E. Pavella and Ruiz-Vega, Transient Stability of Power Systems A Unified Approach to Assessment and Control. Springer, 2010.

[29] R. Zárate-Miñano, T. Van Cutsem, F. Milano, and A. J. Conejo, "Securing transient stability using time-domain simulations within an optimal power flow," IEEE Transactions on Power Systems, vol. 25, no. 1, pp. 243-253, 2009. 